\title{
Expectativa de Vida e Gastos Públicos em Saúde
}

\section{Life Expectancy and Public Health Expenses}

\author{
Maria Lecticia de Pelegrini* \\ Janice Dornelles de Castro**
}

\begin{abstract}
Resumo: Estudo sobre a correlação entre expectativa de vida e nível de gastos públicos em saúde. Utiliza a base de dados do Banco Mundial (amostra de 179 países nos anos de 2003 a 2007). O modelo delineado utiliza a expectativa de vida em função do log gastos públicos. Os resultados indicam relação significativa entre as variáveis. Na ausência de gastos públicos em saúde, a expectativa de vida é de apenas 49,67 anos. O aumento no gasto público per capita em saúde tem um impacto positivo, mas não linear, sobre a expectativa média de vida: para cada dólar de gasto em saúde, há um aumento de 3,93 anos. A expectativa de vida tende a crescer com o aumento dos gastos públicos em saúde. No entanto, o impacto na expectativa de vida depende dos níveis de gastos já existentes em cada país. Onde os gastos públicos são baixos, essa relação tende a ser maior. Nos países com altos níveis de gastos, o aumento dos gastos deverá ser ainda maior para que se verifique um impacto significativo na expectativa de vida.
\end{abstract}

Palavras-chave: Economia da saúde. Desenvolvimento econômico. Expectativa de vida.

\begin{abstract}
Study based on the correlation between life expectancy and level of public health expenditures. Uses the database of World Bank (sample of 179 countries from 2003 to 2007). The model designed assumes life expectancy as a function of public expenditures' log. Results indicate the relation between variables is significant. In the absence of public health expenditures the life expectancy is only 49.67 years. The increase of public expenditures in the health per capita has a positive, but not linear, impact on life expectancy: for each dollar spent on health, there is a rise of 3.93 years. Life expectancy tends to grow as public health expenditures rises. However, the impact in life expectancy depends on current levels of public health expenditures in each country. Where the level of public expenditure is low, such relation tends to be higher. In countries with high levels of expenditures, spending

\footnotetext{
* Doutoranda em Economia da Universidade Federal do Rio Grande do Sul (UFRGS) E-mail: mlmpelegrini@gmail.com

** Professora doutora da Faculdade de Ciências Econômicas da Universidade Federal do Rio Grande do Sul (UFRGS).E-mail: janice.castro@ufrgs.br
} 
should be even higher in order to attain a significant impact on life expectancy. It is highlighted the GNP is an insufficient index to assess welfare.

Keywords: Health economics. Economic development. Life expectancy.

JEL Classification: I18; I10; O1.

\section{Introdução}

O desenvolvimento econômico exerce efeitos importantes na condição de saúde da população; portanto, países com melhores condições de renda tendem a exibir melhores indicadores de saúde. Todavia, o processo inverso, ou seja, a melhora da situação econômica em virtude de um melhor nível de saúde é ainda uma associação não completamente compreendida. Desde 1948, com a Declaração dos Direitos Humanos, a saúde passou a ser tratada como um direito a ser assegurado às pessoas; o desafio atual, no entanto, é assegurar esse direito. A desigualdade em saúde parece estar relacionada aos diferentes níveis de gasto em saúde de cada país. O relatório de Macroeconomia e Saúde (WHO, 2001) demonstra que, em países ricos, onde vivem em torno de 891 milhões de habitantes, o gasto anual médio em saúde chega a US\$25.730,00 e a expectativa de vida é de 78 anos. No entanto, em países pobres, onde estão 1.777 milhões de habitantes, o gasto anual médio com saúde é de apenas US\$38,00 e a expectativa de vida de 59 anos. O menor gasto público com saúde nos países pobres agrava a condição de miséria e morte precoce, interferindo diretamente na expectativa de vida.

A renda é um importante indicador da riqueza, mas não é o único determinante da qualidade de vida da população de um país. A disponibilidade de serviços públicos na área de educação e saúde são instrumentos poderosos utilizados para a redução da desigualdade (OCDE, 2008).

A associação entre aumento de renda e melhores condições de saúde está amplamente descrita na literatura; contudo, poucos estudos demonstram existir uma relação entre melhores condições de saúde e o aumento do crescimento ou desenvolvimento econômico. Lewis (2010), por exemplo, defende que essa relação pode ser estabelecida e que existem sinergias a serem exploradas, por isso defende que a investigação sobre o impacto dos gastos públicos em saúde deveria receber mais destaque nas agendas de pesquisa da área.

Neste estudo buscamos identificar a correlação existente entre gasto público com saúde e a expectativa de vida em alguns países selecionados com o propósito de contribuir para essa discussão. 


\section{Referencial Teórico}

"Saúde e Desenvolvimento Econômico" foi um dos temas tratados na primeira Conferência Mundial de Serviços de Saúde em 1962. Nessa ocasião, foram utilizados os preceitos da teoria do capital humano para discutir a necessidade de investimentos em serviços de saúde como forma de aumentar a produtividade humana e, consequentemente, melhorar o desempenho econômico. Nesse caso, o capital humano representa o estoque de atributo intrínseco aos indivíduos que deve ser visto como uma forma de investimento, ou seja, um trabalhador com saúde produz mais e melhor.

Descreve-se a produtividade como "a arma secreta do capitalismo", cujo aumento constitui a força propulsora para que as empresas melhorem desempenho e adquiram vantagens comparativas. Nessa medida, o investimento na saúde e no bem-estar dos trabalhadores é fundamental, pois as condições de saúde são capazes de afetar a qualidade e capacidade de produção. Assim, uma melhor saúde proporcionaria o aumento da disposição do trabalhador e também de produtividade em suas atividades (SCHULTZ, 1961, 1987). Na ausência de doenças e limitações físicas, o trabalhador seria capaz de executar um maior número de tarefas no trabalho, além de ter mais disposição para o lazer. Além disso, com o aumento de sua expectativa de vida, o trabalhador torna-se mais rentável, dado que o investimento realizado na sua formação gera efeitos durante um período de tempo maior.

O trabalho de Amartya Sen $(1999,2000,2002,2003)$ propõe uma nova concepção de bem-estar afirmando que esse deve ser buscado independentemente do que possa gerar em termos econômicos. Estar livre de doenças e do analfabetismo é relevante para a dignidade, para a felicidade e para a autoestima. Trata-se de um avanço na discussão da concepção de capital humano, na medida em que estabelece que "[...] os seres humanos não são apenas meios de produção (mesmo que eles se destaquem nesse aspecto), mas também o fim do exercício" (SEN, 2003, p. 36).

Essa dualidade da condição humana - por um lado, agentes e beneficiários do progresso e, por outro lado, meios de produção de riquezas econômicas - leva a uma confusão entre o que são "meios" e "fins" do desenvolvimento. Como consequência, prioriza-se a definição de políticas públicas focadas em objetivos como o aumento da renda enquanto se negligenciam outros aspectos igualmente relevantes, como saúde e educação.

Sen (2000) questiona a ênfase dada à renda como único meio de alcançar o bem-estar ${ }^{1} \mathrm{e}$ argumenta que há outras formas para a promoção do desenvolvi-

A abordagem das capacitações fundamentada por Sen (2000) destaca a pluralidade de dimensões que integra a avaliação do bem-estar e critica os enfoques que a reduzem a uma única dimensão, representada pela satisfação e utilidade que a renda e/ou outros recursos e bens podem 
mento e das potencialidades humanas, especialmente aquelas concernentes aos direitos e às oportunidades, que denominou como "liberdades como meios":

a) liberdade política, para votar e eleger, opinar e reclamar;

b) facilidades econômicas, ou o acesso a recursos, à troca, ao consumo;

c) oportunidades sociais, obtida pela educação, boa saúde e capacidade de interagir com os outros membros da sociedade;

d) garantia de transparência, que permite exercer a confiança;

e) segurança protetora, capaz de reduzir a vulnerabilidade e riscos frente a guerras, epidemias e incerteza de eventos externos.

Em sua manifestação sobre a questão da saúde e desenvolvimento, reforça que o aprimoramento da saúde das pessoas deve ser aceito como um dos principais objetivos do processo de desenvolvimento. Porém, esse reconhecimento não é tão simples, pois coloca em discussão se melhores condições de saúde são consequência do desenvolvimento econômico, ou se a melhor condição de saúde é parte do processo que permite o desenvolvimento econômico (SEN,1999).

Para o autor, é necessário retomar a discussão acerca da concepção de desenvolvimento e propõe a utilização de um enfoque mais amplo, que considere a questão sob a perspectiva da "forma de levar a vida" e não apenas do acúmulo de riqueza. A utilidade da riqueza está naquilo que ela nos permite fazer, nas liberdades substantivas que nos ajudam a realizar, incluindo a liberdade de viver muito e viver bem. Mas essa relação não é exclusiva (já que há outras influências significativas em nossas vidas que não a riqueza), nem uniforme (já que o impacto da riqueza em nossas vidas varia de acordo com outras influências). Reconhecer o papel crucial da riqueza nas condições e qualidade de vida torna-se tão importante quanto compreender a importância crucial da saúde para isso. Portanto, a concepção adequada de desenvolvimento precisa ir muito além da avaliação do acúmulo de riqueza representada pelo crescimento do Produto Nacional Bruto ou outras variáveis relacionadas à renda.

O trabalho de Sen coloca em discussão os efeitos nefastos das desigualdades em saúde para o desenvolvimento humano. Além desse, autores como Anand e Ravalion (1993), Marmot (2002), Thomas e Frankenberg (2002), Alleyne (2002) e organismos internacionais, como a Organização Mundial da Saúde e o Banco Mundial, em seus estudos buscam explicar e indicar caminhos para obter melhores resultados na relação entre economia e saúde.

Desde 1970, os economistas da saúde discutem e estudam a relação entre a saúde da população e o rendimento nacional. Preston (1975) é um dos pioneiros, com seu trabalho conhecido como a "Curva de Preston". A partir de uma seleção

oferecer ao homem. A abordagem das capacitações surge como crítica a teorias como utilitarismo, a teoria Rawlsiana dos bens primários, necessidades básicas, visões de liberdades formais e libertarismo e perspectivas de renda. Sobre essas teorias, salienta as dificuldades e o restrito espaço para o indivíduo obter as informações suficientes e assim estabelecer juízos avaliativos. 
cruzada de países, em uma série temporal de dados de 1900, 1930 e 1960, demonstra a relação entre expectativa de vida e renda nacional per capita.

Estabelece-se uma forte associação entre PIB e saúde com o estudo de Anand (1993). Ele afirma que o incremento de despesas públicas com políticas sociais, especialmente em saúde, com intervenções de baixo custo e resultados de grande escala, tornam os investimentos em saúde um instrumento promissor para os países em desenvolvimento.

No relatório de Macroeconomia e Saúde (WHO, 2001), Bloom e Canning examinam o papel da saúde na geração de bem-estar econômico e discutem qual a possibilidade de melhores condições de saúde contribuírem para o aumento da expectativa de vida, independente do aumento de renda. Demonstram que a situação de saúde melhora mesmo que a renda tenha se mantido fixa. Os autores afirmam que mais de $75 \%$ dos ganhos em saúde decorrem da elevação de gastos em saúde, e menos de $25 \%$ do movimento é consequência do enriquecimento dos países. Para os autores, essa constatação reforça a ideia de que intervenções diretas em saúde são mais eficientes para melhorarem a saúde da população.

As conclusões do Relatório de Macroeconomia e Saúde apontam que deve ser meta prioritária para o desenvolvimento econômico melhorar a situação de saúde. Indicam também que a importância do investimento em saúde tem sido subestimada, não só pelos analistas, mas pelos governos e pela comunidade internacional de doadores, defendendo que maiores investimentos nessa área poderão trazer grandes benefícios sociais.

Estudos como o de Thomas e Frankenberg (2002), por outro lado, discutem o papel da nutrição e da saúde para a produtividade, reforçando as evidências de que há uma forte associação entre melhores condições de saúde e crescimento econômico. No entanto, consideram que o nexo causal possa ser em ambas as direções, ou seja, com maior rendimento os indivíduos investem mais em capital humano, ou seja, em melhores dietas, saneamento e cuidados de saúde. E, por outro lado, sendo o trabalhador mais saudável e menos suscetível às doenças, é mais produtivo e obtém rendimento maior.

Nordhaus (2003) demonstra que os ganhos em saúde têm sido comparáveis, e até ultrapassam os ganhos de renda em muitos países. Isso reforça sua conviç̧ão de que, embora essas diferenças de renda tenham sido persistentes ao longo dos últimos 50 anos, há um crescimento maior da expectativa de vida.

As grandes desigualdades em saúde apresentam um desafio para o mundo: a expectativa de vida nos diferentes países pode variar em até 48 anos, conforme enfatiza Marmot (2005). A origem de parte dessas desigualdades em saúde é determinada socialmente, e são significativas, tanto para agravos transmissíveis quanto para não transmissíveis. 
Como destaca o trabalho de Spence e Lewis (2009), publicado no relatório da Comissão de Desenvolvimento do Banco Mundial, a saúde é uma fonte direta de bem-estar humano, mas, ao mesmo tempo, um importante instrumento para elevar os níveis de renda. A discussão proposta pelos autores é de quais mecanismos podem afetar a saúde e a renda, analisando questões como:

a) a produtividade do trabalhador,

b) a estrutura da educação infantil,

c) a poupança e o investimento,

d) o impacto da carga de doenças e a expectativa de vida, e

e) o comportamento prospectivo do ciclo de vida.

A Organização mundial da Saúde (OMS) propôs a elaboração e a implantação das Contas Satélites em Saúde. O objetivo torna-se a unificação das informações para criar a possibilidade de comparar gastos com saúde. O Boletim do Sistema Nacional de Contas, de abril de 2010, divulga os achados da pesquisa sobre o financiamento dos sistemas de saúde e demonstra a enorme desigualdade entre a distribuição da população, a expectativa de vida ajustada (DALY) e os gastos com saúde (WHO, 2010a).

Os países da OCDE, que representam menos de $20 \%$ da população, gastam mais de $80 \%$ dos recursos mundiais em saúde, ou ainda, 11,2\% dos seus PIBs, enquanto que nos países africanos e do sudeste asiático (que representam aproximadamente $40 \%$ da população mundial), os gastos representam 4,1\% do PIB (OCDE, 2010).

A análise da situação epidemiológica demonstra a mesma desigualdade e pode ser traduzida pela expectativa de vida. Os países mais ricos são menos populosos, a utilização de recursos de saúde é menor e, ainda assim, possuem uma carga de doenças menor que os países mais pobres, com populações maiores (WHO, 2010b).

O papel dos Estados na constituição de sistemas de saúde como conhecemos tem início no final da primeira metade do século XX, fortemente influenciados pelo Welfare State na Europa, sendo dois os modelos mais importantes: o Seguro Social alemão (Plano Bismark) e os Sistemas Universalistas inglês (Beveridge). Essas formas de organização são gradativamente adotadas em todo o continente europeu, atendendo a especificidades de cada país, mas sempre com grande nível de investimento público. Esses dois modelos servem, também, de exemplo para a formação de sistemas de saúde em todas as partes do mundo.

O papel dos Estados não é uniforme e encontramos diversas possibilidades de financiamento, organização e provimento dos cuidados de saúde. A forma de acesso aos cuidados de saúde é apontada como um dos fatores capazes de piorar a condição de saúde das pessoas. A existência de barreiras, como o pagamento, ou a necessidade de buscar no mercado os serviços de saúde (out-of-pocket), pode 
agravar ainda mais a condição de miséria e morte precoce, interferindo diretamente na expectativa de vida.

\section{Materiais, Métodos e Resultados}

Este estudo utiliza a base de dados do Banco Mundial. A amostra consiste de $179^{2}$ países com dados para os anos de 2003 a 2007. As variáveis selecionadas são expectativa de vida e gastos públicos em saúde. Para cada país, utiliza-se a média de cada uma das variáveis no período. A variável gasto público em saúde per capita é calculada através da informação do gasto total em saúde dividido pela população.

Para investigar a capacidade do gasto público em saúde determinar a expectativa de vida, delineia-se um modelo sob a hipótese de que as variáveis expectativa de vida e gastos públicos em saúde não apresentam uma correlação linear. Os valores para as análises são gerados com o programa Eviews, a partir da seguinte especificação:

$$
y=\beta_{0}+\beta_{1} \log (x)+\varepsilon
$$

Onde: y é a expectativa de vida, x é o gasto público per capita em saúde, e $\mathcal{E}$ o erro com média zero.

2 Afeganistão, Albânia, Argélia, Angola, Argentina, Armênia, Austrália, Áustria, Azerbaijão, Bahamas, The Bahrain, Bangladesh, Barbados, Belarus, Bélgica, Belize, Benin, Butão, Bolívia, Bósnia e Herzegovina, Botsuana, Brasil, Brunei Darussalam, Bulgária, Burkina Faso, Burundi, Camboja, Camarões, Canadá, Cabo Verde, República Central Africana, Chade, Chile, China, Colômbia, Camarões, Congo, Dem. Rep. do Congo, Rep. Costa Rica, Costa do Marfim, Croácia, Cuba, Chipre, República Checa, Dinamarca, Djibuti, República Dominicana, Equador, Egito, Emirados Árabes Unidos, El Salvador, Guiné Equatorial, Eritreia, Estônia, Etiópia, Fiji, Finlândia, França, Gabão, Gâmbia, Geórgia, Alemanha, Gana, Grécia, Granada, Guatemala, Guiné, Guiné-Bissau, Guiana, Haiti, Honduras, Hungria, Islândia, Índia, Indonésia, Irã, República Islâmica do Iraque, Irlanda, Israel, Itália, Jamaica, Japão, Jordânia, Cazaquistão, Quênia, Coreia, República da Kuwait, Quirguistão, Laos, Letônia, Líbano, Lesoto, Libéria, Líbia, Lituânia, Luxemburgo, Macedônia, ARJ Madagascar, Malaia, Malásia, Maldivas, Mali, Malta, Ilhas Marshall, Mauritânia, Maurícias, México, Micronésia, Fed. Estados da Moldávia, Mônaco, Mongólia, Montenegro, Marrocos, Moçambique, Namíbia, Nepal, Países Baixos, Nova Zelândia, Nicarágua, Níger, Nigéria, Noruega, Omã, Paquistão, Palau, Panamá, Papua Nova Guiné, Paraguai, Peru, Filipinas, Polônia, Portugal, Qatar, Romênia, Federação Russa, Ruanda, Samoa, San Marino, São Tomé e Príncipe, Arábia Saudita, Senegal, Sérvia, Seychelles, Serra Leoa, Cingapura, Eslováquia, Eslovênia, Ilhas Salomão, África do Sul, Espanha, Sri Lanka, St. Lucia, St. Vincent e Granadinas, Sudão, Suriname, Suazilândia, Suécia, Suíça, República Árabe da Síria, Tajiquistão, Tanzânia, Tailândia, Timor-Leste, Togo, Tonga, Trinidad e Tobago, Tunísia, Turquia, Turcomenistão, Uganda, Ucrânia, Reino Unido, Estados Unidos, Uruguai, Uzbequistão, Vanuatu, Venezuela, Vietnã, Zâmbia, Zimbábue. 
Quadro 1- Saída do Eviews para o modelo: expectativa de vida em função dos gasto público per capita para 179 países no período de 2003 a 2007

\begin{tabular}{|c|c|c|l|l|}
\hline $\begin{array}{c}\text { Dependent Varia- } \\
\text { ble: EXP_VIDA }\end{array}$ & & & & \\
\hline $\begin{array}{c}\text { Method: Least } \\
\text { Squares }\end{array}$ & & & & \\
\hline $\begin{array}{c}\text { Date: } 11 / 07 / 10 \\
\text { Time: } 17: 19\end{array}$ & & & & \\
\hline Sample: 1 180 & & & & \\
\hline $\begin{array}{c}\text { Included observa- } \\
\text { tions: } 179\end{array}$ & & & & \\
\hline & Coefficient & Std. Error & t-Statistic & Prob. \\
\hline Variable & & & & 0 \\
\hline C & 49,67868 & 1,302202 & 38,14976 & 0 \\
\hline GASTO_PUB_LOG & 3,931222 & 0,260384 & 15,09776 & 67,70341 \\
\hline & & & & 10,49357 \\
\hline R-squared & 0,562901 & $\begin{array}{c}\text { Mean dependent } \\
\text { var }\end{array}$ & & 6,728551 \\
\hline Adjusted R-squared & 0,560431 & S.D. dependent var & & 6,764164 \\
\hline S.E. of regression & 6,957233 & $\begin{array}{c}\text { Akaike info cri- } \\
\text { terion }\end{array}$ & & 227,9425 \\
\hline Sum squared resid & 8567,347 & Schwarz criterion & & 0 \\
\hline Log likelihood & $-600,2053$ & F-statistic & & \\
\hline Durbin-Watson stat & 1,94159 & Prob(F-statistic) & & \\
\hline
\end{tabular}

Fonte: Elaboração própria a partir da base de dados do Banco Mundial.

O valor de $R^{2}$ de 0,5629 com o p-value permite rejeitar a hipótese nula para ambos os parâmetros. Indica que a relação entre as variáveis é significativa. Isso implica que a expectativa de vida tende a crescer com o aumento dos gastos públicos em saúde.

Quando $\log (\mathrm{x})=0, \beta_{0}=49,67$, ou seja, uma expectativa de vida de 49,67 anos. Há uma significativa correlação entre as variáveis.

Quando $\mathrm{x}=$ US\$1, a expectativa média de vida é 3,93. Portanto, para cada dólar gasto em saúde, há um aumento de 3,93 anos na expectativa de vida. log $(\mathrm{x})=0$ (isto é, quando $\mathrm{x}=$ US $\$ 1$ ), a expectativa média de vida fica em 49,67 anos.

A correlação pode também ser visualizada no Gráfico 1. 
Gráfico 1- Expectativa de vida e log (gastos públicos per capita) de 179 países

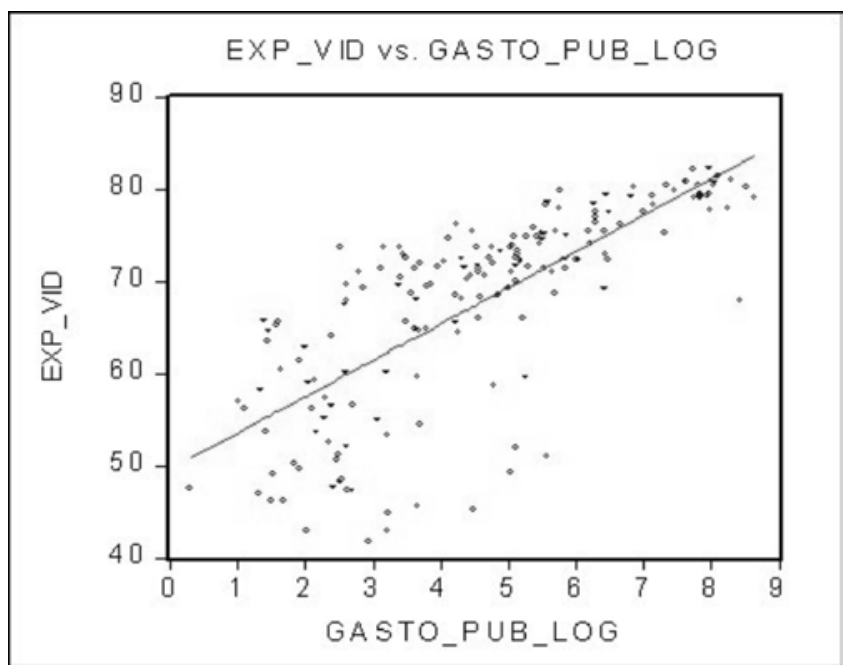

Fonte: Elaboração própria a partir da base de dados do Banco Mundial.

O aumento de gastos de saúde não demonstra correlação direta com a expectativa de vida, mas o gasto precisa aumentar para que se aumente também a expectativa de vida, considerando-se os níveis de gastos já existentes. Para países com gastos baixos, essa relação é maior (de 1 para 1). No entanto, em países que já possuem gastos elevados com saúde, este aumento deverá ser ainda maior para que o impacto venha a se tornar significativo.

\section{Considerações Finais}

Os resultados encontrados neste estudo são coerentes com os de outros trabalhos identificados na literatura sobre o mesmo tema. O relatório estatístico da OMS (WHO, 2010b) apresenta uma análise de regressão feita entre a média de expectativa de vida em anos e a média total de gastos com saúde no ano de 2007 para as sete regiões da OMS. ${ }^{3}$

As conclusões indicam que a expectativa de vida aumenta com o aumento da despesa em saúde per capita total. No entanto, para os países com os níveis de despesa em saúde per capita mais elevados, estão associados apenas modestos ou nenhuma melhoria na expectativa de vida com aumento de despesa em saúde. Além disso, uma parcela dos países alcançou altos níveis de expectativa de vida, apesar de realizar despesas relativamente menores.

São elas: AFR: países africanos; SEAR: países da Ásia Oriental Sul; EUR: países da Europa: EMR: países do Mediterrâneo Oriental; AMR: países das Américas; WPR: região oeste do Pacífico. 
Em quase um quarto dos países, as despesas com saúde, em 2007, foi inferior a US\$100 per capita - a maioria destes países está localizada na Região Africana da OMS, sendo que três quartos deles tinham uma esperança de vida de 60 anos ou menos. Os países com maiores níveis de despesa per capita com a saúde são principalmente os da região das Américas e da região Europeia da OMS.

O relatório de Macroeconomia e Saúde (WHO, 2001) apresenta os resultados de regressão feita entre Log (PIB per capita) e Log (gastos públicos per capita) em 167 países em 1997. Neste estudo, a variável "gastos com saúde" foi determinada principalmente pela renda nacional. Os países mais pobres exibem um padrão de gasto muito baixo se comparados com os países ricos, e não dispõem de mais recursos para satisfazer as necessidades básicas de saúde de suas populações. Nesses casos, gastar US\$ 40 per capita pode representar mais de $10 \%$ do PIB. Apesar dessa constatação, a recomendação da comissão é de aumentar o gasto com saúde através de recursos internos, especialmente os orçamentários, mesmo sabendo que a capacidade de gerar maiores receitas públicas para a saúde difere de país para país, essencialmente em função das suas estruturas econômicas e das capacidades de arrecadação tributária. Em geral, os países mais pobres mobilizam uma parcela menor do PIB em receitas fiscais, uma média de 14\% do PIB, em comparação com 31\% do PIB nos países de alta renda.

Por outro lado, os dados publicados pela OCED (2010) mostram que o crescimento anual das despesas per capita com saúde tem sido maior que o crescimento do PIB per capita no período analisado de 2000-2008, indicando que os países de alta renda necessitam, cada vez mais, de maiores investimentos em saúde. No entanto, há situações em que, embora os países tenham PIB per capita menor (caso da França em relação aos Estados Unidos), a sua esperança de vida ao nascer é maior, e essa vantagem tem aumentado ao longo dos anos, passando de menos de seis meses em 1960 para quase dois anos em 2006.

\section{Referências}

ALLEYNE, G. A. O. Equity and the Goal of Health for All. Revista Panamericana de Salud Pública, Washington, DC, v. 11, n. 5-6, May/Jun. 2002.

ANAND, S; RAVALLION M. Human development in poor countries: on the role of private incomes and public services. Journal of Economic Perspectives, Nashville, US, v. 7, n. 1, p. 133-150, Winter 1993.

BANCO MUNDIAL. Data: Helth. 2012. Disponível em: < http://data.worldbank.org/topic/ health >. Acesso em: 5 fev. 2012

BRASIL. Presidência da República. Casa Civil. Lei no 8.080, de 19 de setembro de 1990. Dispõe sobre as condições para a promoção, proteção e recuperação da saúde, a organização e o funcionamento dos serviços correspondentes e dá outras providências. 1990. Disponível em: <www.planalto.gov.br/ccivil_03/Leis/L8080>. Acesso em: 4 de junho de 2010 
GUARAJATI, D. Econometria básica. Rio de Janeiro: Elsevier, 2006.

HILL, C. Econometria. São Paulo: Saraiva, 2010.

LEWIS, M. Investimento em saúde e crescimento econômico. 2010. Disponível em: <http:// www.growthcommission.org/storage/cgdev/documents/lewisfinasppresentation $>$. Acesso em: 9 de maio de 2010

MARMOT, M. Social determinants of health inequalities. The Lancet, London, GB, v. 365, n. 9464, p. 1099-1104, Mar. 2005.

ORGANIZATION FOR ECONOMIC CO-OPERATION AND DEVELOPMENT - OECD. Growing Unequal? Income distribution and poverty in OECD countries. Geneva: OECD 2008.

. Source OECD Health Data. 2010. Disponível em: < http://www.oecd.org/health/ healthdata >. Acesso em: 9 set. 2010

THOMAS, D.; FRANKENBERG, E. Health, nutrition and prosperity: a microeconomic perspective. Bulletin World Health Organization, v. 80, n. 2, p. 106-113, 2002.

STIGLITZ, J.; SEN. A.K; FITOUSSI, J. P. Report by the Commission on the Measurement of Economic performance and Social Progress. 2009. Disponível em: <http://www.stiglitz-senfitoussi.fr/en/index.htm > . Acesso em: 12 nov. 2010

SCHULTZ, T. W. Investment in Human Capital. The American Economic Review, Nashville, US, v. 51, n. 1, p. 1-17, Mar. 1996.

. Investindo no povo: o significado econômico da qualidade da população. Rio de Janeiro: Forense Universitária, 1987.

SEN, A. K. Health in development. Bulletin of the World Health Organization, New York, US, v.77, n. 8, p. $619-623,1999$.

. Desenvolvimento como liberdade. São Paulo: Cia. das Letras, 2000.

. Human capital and human capability. In: FUKUDA-PARR, S.; KUMAR, A. K. S. (Ed.). $\overline{R e a d i n}$ s in human development: concepts, measures and policies for a development paradigm. New York: United Nations Development Programme, 2003.

. ¿Por qué la equidad en salud? Revista Panamericana de Salud Pública, Washington,

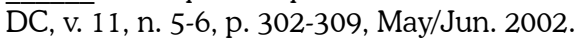

SPENCE, M.; LEWIS, M. Saúde e desenvolvimento. 2009. Disponível em: < http://growthcommission.org > . Acesso em: 20 set. 2010.

WORLD HEALTH ORGANIZATION - WHO. National Health Accounts. Policy Highlight, n.1, Apr. 2010. Disponível em: < http://www.who.int/nha/use/highlights/en/index.html >. Acesso em: 20 set. 2010.

. World Health Organization World health statistics. 2010. Disponível em: < http://www. who.int/whosis/whostat/2010/en/index.html >. Acesso em: 20 set. 2010.

. Macroeconomics and health: Investing in health for economic development: Report of the Commission on Macroeconomics and Health. Geneva: World Health Organization , 2001.

Recebido em: 10/02/2012.

Aceito em: 04/06/2012. 